\title{
On the Importance of Correct Inclusion of Capillary Pressure in Reservoir Simulation
}

\author{
* Ba T., ** Haoy L. B. \\ * Norsk Hydro E \& P Research Centre, Bergen, Norway \\ ** Student, University of Bergen, Norway
}

\begin{abstract}
Copyright 1985, Steering Committee of the European IOA - Symposium.
This paper wes presented at the 8th. Europoan IOR - Symposium in Vienna, Austria, May 16 - 17.1996

This paper wess selected for presentation by the Stoerind Committeo, following roviow of information contained in an abstract submitted by the author(s). The paper, as presented has not been reviowed by the Steering Committeo.
\end{abstract}

\begin{abstract}
Capillary pressure is frequently omitted from large scale reservoir simulation, rationalized by arguments that may not always be valid. A number of papers have shown that correct modelling of capillary pressure may be as important for the simulation result as properly accounting for relative permeabilities. Especially in strongly heterogeneous models it is important to study the sensitivity to all petrophysical parameters before simplifications are introduced in the simulation model.
\end{abstract}

In a previous paper from Norsk Hydro [1] it was demonstrated and explained why stochastic oil/water relative permeabilities (varying from cell to cell) in many cases can be replaced with one pair of "average" relative permeabilities without significantly changing the simulation results. Our current study indicates that a similar procedure is not always valid for capillary pressure.

A number of simulations exemplifies the effects of stochastic capillary pressure in various displacement regimes in a realistic geostatistical reservoir model:

- Gravity dominated water/oil displacement

- Viscous dominated water/oil displacement

- Varying water/oil mobility ratios

- Including/excluding capillary pressure

- Stochastic vs. constant capillary pressure

- Grid sensitivities

Even if the simulation results can be explained by well understood physical theory, our study emphasizes that the net effect of capillary pressure on the oil production curve is in general difficult to predict without numerical simulation on a fine grid: Front smearing tends to give earlier water breakthrough while suppressing of channelling/fingering will have the opposite effect. These true physical effects can be obscured by the numerical diffusion introduced by the finite difference scheme applied in the reservoir simulator.

\section{INTRODUCTION}

Water/oil flow in a reservoir under production is governed by the interaction of viscous, gravity, and capillary forces. Under the assumption of incompressible, isothermal, immiscible water-oil displacement; the volume conservation equation related to the location $\mathbf{x}$ can be expressed as

$$
\begin{aligned}
& \phi \frac{\partial S_{w}}{\partial t}+\nabla \cdot\left\{f\left(S_{w}\right) \mathbf{u}(\mathbf{x})\right\}+\nabla \cdot \mathbf{K} \lambda_{\odot} f\left(S_{w}\right) \\
& \left\{\left(\rho_{w}-\rho_{o}\right) g \mathbf{k}+\nabla P_{c}\right\}=q(\mathbf{x})
\end{aligned}
$$

where the source/sink term $q(\mathbf{x})$ is equal to zero everywhere outside the wells. The second, third, and fourth term represent the flow caused by the viscous, gravity, and capillary pressure, respectively.

A necessary condition for obtaining a contribution from the capillary term is that $\nabla P_{c}=\frac{d P_{c}}{d S_{w i}} \nabla S_{u} \neq$ 0 , which implies that water saturation must vary in space and capillary pressure must vary with saturation. There are three situations where water (or oil) saturation can change abruptly if no capillary forces are present:

- Across a viscous generated shock front, described by Buckley-Leverett theory

- Gravity segregation of phases with different density

- Heterogeneity induced separation of phases

In these cases capillary pressure tend to dampen the saturation shocks, leading to "transition zones" with smoothly varying saturation. However, if capillary pressure is the dominating force, the limiting equilibrium situation is constant capillary pressure throughout the reservoir. Since capillary pressure correlates strongly with permeability and porosity, regions of different rock properties may then have different saturations.

In a completely homogeneous reservoir, where all the rock and fluid parameters are independent of position, it is possible to introduce a set of dimensionless 
numbers which completely determines the two phase flow behavior. This problem was solved already by Rapoport [2] in 1955.

In this study we shall restrict ourselves to a two-dimensional rectangular reservoir with horizontal length $L$ and vertical thickness $H$. The reservoir has an injection well in the leftmost column and a producing well in the rightmost column, and is produced with a constant (reservoir) injection/production rate $q$. which corresponds to a constant horizontal total Darcy velocity $u_{x}$. Then, four dimensionless numbers essentially determines the flow:

$$
\begin{array}{ll}
\text { Shape factor: } & R_{l}=\frac{L}{H} \sqrt{\frac{k_{x}}{k_{x}}} \\
\text { Gravity number: } & N_{g}=\frac{\left(\rho_{w}-\rho_{0}\right) g H k_{x}}{L u_{x} \mu_{0}}=\frac{\Delta \rho g H}{\Delta p} \\
\text { Capillary number: } & N_{c}=\frac{p_{i}^{*} k_{x}}{L u_{x} \mu_{0}}=\frac{p_{c}^{*}}{\Delta p} \\
\text { Endpoint mobility ratio: } & M=\frac{k_{r w}\left(S_{o r w}\right) / \mu_{w}}{k_{r o}\left(S_{w}\right) / \mu_{0}}
\end{array}
$$

While Shape factor (Aspect ratio) and Gravity number characterize single phase flow, Capillary number and Endpoint mobility ratio include two phase flow effects.

Zhou, Fayers, and Orr [3] among others have proposed a modification of the gravity and capillary scaling groups:

$$
\begin{aligned}
& N_{g v}=N_{g} R_{l}^{2}=\frac{T_{\phi}}{T_{g v}} \\
& N_{c v}=N_{c} R_{l}^{2}=\frac{T_{A}}{T_{c v}}
\end{aligned}
$$

Here, $T_{h}$ is the time for oil (one-phase) to flow across the reservoir in the horizontal direction, caused by the viscous force (horizontal pressure drop). $T_{g v}$ and $T_{c v}$ are the times for oil to flow across the reservoir in the vertical direction caused by the gravity and capillary force, respectively.

Condition for viscous dominated flow:

$$
N_{g v}, N_{e v}<<1.0
$$

Condition for gravity dominated flow:

$$
N_{g v} \gg>1.0 \gg N_{c v}
$$

Condition for capillary dominated flow:

$$
N_{e v}>1.0>N_{g v}
$$

During the last 15 years a number of papers have extended the dimensionless or inspectional analysis to heterogeneous reservoirs. The first simple approximation is to substitute the constant horizontal and vertical permeabilities $\left(k_{x}, k_{z}\right)$ with their averaged counterparts; calculated by analytical formulas or simulated (- as explained by Grindheim and Aasen[4]). However, this approach may lead to wrong conclusions in cases with strong local variations in the flow properties.
Yokohama and Lake [5] proved that capillary crossflow in a layered reservoir (with perméability contrasts and communication between layers) is correlated with the number $N_{c v}$; the larger the value of $N_{c v}$, the less is the tendency for fast channeling through the high permeable layers and the longer is the oil production plateau. But capillary pressure may also have an opposite effect. Smearing of the main saturation shock front may lead to faster water breakthrough. For a constant aspect ratio, large values of $N_{c}$ enhance this dispersive effect.

In a realistic geologic heterogeneity model there are mixtures of more or less spatial correlated heterogeneities on different scales, leading to a very tortuous flow. Then, there is no more a constant horizontal Darcy velocity $u_{x}$ and formulas based on average properties may break down. $\mathrm{Li}$ and Lake [6] have generalized the previous work to stochastic reservoir models with permeability variation and spatial correlation on three scales; local, zonal and global. Each scale has its own flow behavior. Local and global heterogeneities cause fingering and dispersive flow, while zonal heterogeneity causes channeling.

Langlo and Espedal [7] have studied the interplay between the capillary pressure and heterogeneity generated saturation fingers. If the scale of capillary diffusion is smaller than the scale of the heterogeneity, capillary diffusion is insignificant compared with the heterogeneous fingering. If the capillary diffusion is on the same scale as the heterogeneity, the heterogeneous fingering is reduced. For even larger scale capillary diffusion the heterogeneous fingering can be neglected.

A very important important capillary effect is highlighted in a number of papers from Heriot-Watt University $[8,9,10]:$ In certain small scale $(\mathrm{mm} / \mathrm{cm} / \mathrm{dm})$ periodic heterogeneity patterns, significant amounts of mobile oil may be trapped in the high permeability parts, leading to a much higher remaining oil saturation than what is predicted from viscous dominated core floods. This mechanism has been demonstrated both with fine grid numerical simulations and experimental work. The implication for field scale numerical simulation is that small scale capillary trapping must be included in the (pseudo) saturation dependent functions applied in the large grid. This is an example of a general problem in numerical simulation: Physical effects on a scale smaller than the grid block dimensions can only be accounted for by pseudo parameters.

In this work we concentrate on demonstrating/ quantifying capillary effects in simulation models with grid block dimensions $4-20 \mathrm{~m}$ in the $\mathrm{x}$-directions and $0.4-2 \mathrm{~m}$ in the z-direction. Angert and Begg [11] and Fitzmorris, Kelsey and Pande [12] have performed similar studies with capillary pressure included in their models. Both papers conclude that capillary pressure may have significant effects both on water breakthrough and total oil recovery. The production 
profile can be positively or negatively affected, depending on the heterogeneity model and the balance of forces.

\section{AIM OF STUDY}

An earlier study from Norsk Hydro [1] concerning effects' of stochastic relative permeabilities in reservoir simulation, demonstrated that in many cases the oil production profile is not significantly altered if only one pair of average curves is applied throughout the simulation grid. In order to produce a realistic pair of average relative permeability curves one need enough measurements to cover the geological variation; especially it is important to obtain realistic estimates of the mean values of initial water saturation and residual oil saturation since these parameters determine the mobile oil volume. It should be emphasized that the cited result is only valid for simple models covering one depositional unit. The result supports the common praxis of applying one pair of relative permeability curves for each geological unit in fine scale reservoir simulation. However, since pseudo curves are not applied, no effects of heterogeneities on smaller scale than the gridblock dimensions are included.

It was observed that in the experimental dataset there is no significant correlation between absolute permeability and the water/oil shock front velocity. This fact inspired Holden [13] to investigate a generalization of the Buckley-Leverett model to a stochastic 1D porous medium. Essentially he proved that one pair of average relative permeability curves can replace spatially varying relative permeability curves as long as there is no spatial correlation between the curves.

In the previous study capillary pressure was not included in the simulation model. The next step will then be to investigate capillary pressure effects in the simulation results:

- What are the important effects of capillary pressure under various balances of viscous, gravity and capillary forces?

- Capillary pressure is determined by a formula including absolute permeability, porosity, and initial water saturation. What is the implication of substituting varying capillary pressure with one average curve?

- How is capillary pressure affecting the spread in production profiles from several realizations of the same geostatistical model?

- How does grid refinement affect the simulation results?

\section{SIMULATION MODEL}

Our work is a continuation of earlier studies from Norsk Hydro $[1,14]$. Based on measurements on 40 core plugs from a North Sea well in a Distal Mouthbar deposit, a geostatistical model has been developed. A simplified $2 \mathrm{D}$ representation is a rectangle of dimensions $L=720 \mathrm{~m}$ and $H=96 \mathrm{~m}$ where the spatial variability of the various petrophysical variables is governed by a simple Gaussian random field model. The mean and the variance is given for each variable, as well as the range in a common spherical variogram. The correlation lengths in the horizontal and vertical directions are set equal to $L$ and $H / 4$, respectively. Fig. 1 shows the distribution of horizontal permeability for a given realization.

Also experimentally obtained correlations between petrophysical parameters are respected in the model construction. There is a Pearson correlation of 0.7 between horizontal and vertical permeabilities, with a factor 10 between the means. Tables 1 and 2 summarize the parameter variations and correlations. Even within a fixed depositional environment there are large parameter variations. One observes as expected, strong correlations between $\log k_{x}$ and $S_{w i}$, $\log k_{x}$ and $k_{r w}\left(S_{\text {orw }}\right)$. Most parameter pairs, however, are only weakly correlated.

Capillary pressure is given by a deterministic formula obtained from multivariate analysis between logs and core measurements [15]:

$$
P_{c}\left(S_{w}\right)=0.0865 \phi^{-0.906} k^{-0.109} S_{w}^{*-0.744}-0.192
$$

where $S_{w}^{*}$ is normalized water saturation defined by

$$
S_{w}^{*}=\frac{S_{w}-S_{w i}}{1-S_{o r w}-S_{w i}}
$$

When $S_{w}$ tends towards $S_{w i}$ the capillary pressure tends towards infinity. To obtain a finite value for $P\left(S_{w i}\right)$ we define (arbitrarily) $P\left(S_{w i}\right)=P\left(S_{w i}+0.01\right)$

The measured capillary pressure drainage curves are obtained from porous plate experiments. The imbibition curves may differ substantially from the drainage curves, but in this generic study we have chosen to apply the drainage curves also for water displacing oil.

Some simulator runs are performed with constant relative permeability curves obtained by arithmetical averaging of each rel.perm. parameter over the simulation grid. The corresponding capillary pressure is obtained by applying the formula above with mean values of porosity, permeability and initial water saturation.

We have simulated cases with equal oil and water densities at reservoir conditions, and also cases with significant gravity effects $\left(\Delta \rho=0.156 \mathrm{~kg} / \mathrm{m}^{3}\right)$. To study the influence of mobility ratio we have applied $M=1$ and $M=10$.

The gravity numbers in these models are $N_{g v} \approx$ 20 for $M=1$ and $N_{g v} \approx 2$ for $M=10$, indicating strong gravity influenced flow. The corresponding capillary numbers are $N_{c v} \approx 2$ and $N_{c v} \approx 0.2$, indicating substantial effects of capillary pressure. 
Three different cartesian regular grids have been applied: $36 \times 48,180 \times 48$, and $36 \times 240$ When comparing runs with fine and coarse grids, absolute permeabilities are always scaled from the fine to the coarse grid by a single phase simulation method.

When comparing several realizations from the geostatistical model the total pore volume has been scaled in order to maintain identical movable pore volumes.

The simulator applied is commercial software (ECLIPSE) with one-point upstream weighting and fully implicit solution of the system of pressure and saturation equations. The simulator is run with constant and equal injection/production rates corresponding to $5 \%$ pore volume per year. Tables 3 and 4 gives an overview of the various runs with corresponding cumulative oil production at water breakthrough and after 15 years of production.

\section{RESULTS}

The main emphasize is on results with and without capillary pressure and comparison of constant vs. varying capillary pressure. We also consider grid refinement. Table 3 summarizes the different simulation runs and the main production results.

No gravity effects: In order to cancel any gravity effect the oil and water densities (- at reservoir conditions) are assumed identical. These runs are performed on a $180 \times 48$ grid, that is; with grid refinement in the horizontal direction in order to allow for detailed modeling of channeling/fingering. Fig. 2 shows that in all cases there are some differences between the oil production profiles with/without capillary pressure for a period just after water breakthrough. However, there is no significant effect on the total cumulative oil production. For unit mobility ratio there is a 2 years difference in water breakthrough times with constant and variable saturation dependent functions.

Fig. 3 illustrates the effect of (varying) capillary pressure in the case with $M=10$. While there are three pronounced channels without capillary pressure, capillary crossflow "connect" the channels. The effect on the oil production profiles, however, is less than what could be expected from the saturation plots.

Fig. 4 shows saturation plots for the cases with unit mobility ratio, with constant and variable capillary pressure. The plots confirm that after 6 years the water front has not reached the producer when capillary pressure is constant, but with varying capillary pressure there is more dispersion and faster water breakthrough.

Different oil and water densities: In order to obtain a detailed resolution in the vertical direction the grid has $36 \times 240$ blocks. Fig. 5 shows that with sparially constant relative permeabilities there is no effect of capillary pressure when $M=1$. When $M=10$ constant capillary pressure gives later water breakthrough and significantly higher cumulative oil pitoduction than the corresponding case without capiliary pressure.

With varying relative permeabilities, water breakthrough arrives first when capillary pressure is included. For $M=1$ there is also significantly higher cumulative oil production when capillary pressure is not included.

Fig. 6 shows saturation plots for the case with $M=10$, constant relative permeability, with/without (constant) capillary pressure. Without capillary pressure there is a lot of channeling/fingering and not a very pronounced effect of gravity. Capillary crossflow together with gravity segregation change the picture. Now there is a gravity tongue developing, but still with significant channeling behind the primary water front. In both cases there is a high degree of dispersion.

Different Realizations: 5 realizations with the same geostochastical geological model have been simulated. Fig. 7 shows an example (Constant rel.perm., $\Delta \rho>0, M=10$ ) where capillary pressure significantly reduces the variation in the production profiles compared to the similar runs where the capillary pressure is not included. However, this is not a general result. In the case with variable rel.perm., no gravity effects and $M=1$; fig. 8 shows that the variation in the production profiles has not decreased when capillary pressure is included.

Grid Sensitivity: The $180 \times 48$ and $36 \times 240$ grids are reduced to a $36 \times 48$ grid by compressing five and five blocks in the horizontal, respectively the vertical direction.

Comparing results with fine and coarse grids in the horizontal direction, only minor effects are observed regarding the oil production profiles for constant saturation dependent functions. With variable relative permeability and capillary pressure there is always a significant later water breakthrough in the fine grid compared with the coarse grid case (fig. 9). For grid sensitivity in the vertical direction, in those cases where there are differences between the fine and coarse grid simulation results, the fine grid production profiles always show the fastest water breakthrough.

Another observation is that the difference in production profiles between similar fine grid and coarse grid simulation are reduced when capillary pressure is included. Following the arguments in the paper by Lantz [16]; capillary and numerical diffusion add in the applied numerical scheme (one point upstream weighting, implicit solution). However, while numerical diffusion is proportional with the horizontal grid block dimension, capillary diffusion is larger the smaller the grid block size (since the first order approximation of $\nabla P_{c}$ is increased by reduced $\Delta x$ ) 


\section{CONCLUSIONS}

The main conclusion is that capillary pressure may significantly affect the flow behavior. The capillary pressure effect can be much stronger in models with heterogeneities on different scales than in homogeneous or layered systems. The net effect on oil and water production depends on the balance between the capillary diffusion and capillary crossflow.

Observations from our simulation study can be summarized as follows:

* Capillary pressure may have significant effects on simulated oil production profiles even with gridblock dimensions in the $10 \mathrm{~m}$ range.

* Large differences in local saturation distributions do not always show up in the oil production profiles.

* Dimensionless capillary and gravity numbers give "rules of thumb" for the importance of capillary pressure in field scale simulations. Only in the gravity dominated case $\left(N_{g v} \approx 20\right)$ there are no observable effects of capillary pressure on the oil production profile. In all other cases that have been investigated there are differences between runs with/without inclusion of capillary pressure.

* There are significant differences between runs with constant and variable relative capillary pressure, implying that previously obtained results regarding relative permeability variation cannot be extended to capillary pressure.

- Examples show that capillary pressure can both increase and reduce the variation in oil production profiles for a number of realizations from the same geostatistical model.

* The sensitivity to grid coarsening is less with than without capillary pressure included in the simulation model.

\section{ACKNOWLEDGEMENT}

The paper is a part of Norsk Hydro's contribution to the Commission of the European Communities' JOULE programme, subprogramme Energy from Fossil Sources, Hydrocarbons, Reservoir Engineering Project; founded in part by the Research Council of Norway.

\section{NOMENCLATURE}

$\begin{array}{ll}f & \text { fractional flow } \\ g & \text { gravity acceleration } \\ k & \text { absolute permeability } \\ k_{r} & \text { relative permeability } \\ n & \text { Corey exponent } \\ o & \text { oil } \\ o r w & \text { residual oil } \\ w & \text { water } \\ w i & \text { initial water } \\ q & \text { source/sink term } \\ u & \text { scalar velocity }\end{array}$

$\begin{array}{ll}x & \text { horizontal direction } \\ z & \text { vertical direction } \\ H & \text { reservoir thickness } \\ L & \text { reservoir length } \\ M & \text { mobility ratio } \\ N & \text { dimensionless number } \\ P_{e} & \text { capillary pressure } \\ S & \text { saturation } \\ \mathbf{k} & \text { vertical unity vector } \\ \mathbf{K} & \text { permeability tensor } \\ \mathbf{u} & \text { total velocity vector } \\ \mathbf{x} & \text { location vector } \\ \mu & \text { viscosity } \\ \lambda & \text { mobility } \\ \rho & \text { density }\end{array}$

\section{REFERENCES}

[1] Tjølsen, C.B, Damsleth, E. and Bu, T.: The effect of stochastic relative permeabilities in reservoir simulation, J. of Petr. Science \& Eng., Vol. 10 (1994) 273 - 290.

[2] Rapoport, L.A.: Scaling Laws for Use in Design and Operation of Water-Oil Flow Models, Transactions of AIME, Vol 204 (1955) 143 - 150.

[3] Zhou, D., Fayers, F.J. and Orr, F.M.: Scaling of Multiphase Flow in Simple Heterogeneous Porous Media, paper SPE/DOE 27833 presented at the ASME Winter Meeting, New Orleans, Nov. 1993.

[4] Grindheim, A. and Aasen, J.O.: An Evaluation of Homogenization Techniques for Absolute Permeability, Proceedings of First Lerkendal Petroleum Engineering Workshop, Trondheim. 1991, Tapir publ.

[5] Yokoyama, Y. and Lake, L.W.: The Effects of Capillary Pressure on Immiscible Displacement in Stratified Porous Media, paper SPE 10109 presented at 56th SPE Annual Technical Conference, San Antonio, Oct. 1981.

[6] Li, D. and Lake, L.E.: Scaling Fluid Flow Through Heterogeneous Permeable Media, paper SPE 26648 presented at 68th SPE Annual Technical Conference, Houston, Oct. 1993.

[7] Langlo, P. and Espedal, M.S.: Macrodispersion for two-phase, immiscible flow in porous media, Advances in Water Resources, Vol. 30 (1994).

[8] P.S. Ringrose, K.S. Sorbie, P.W.M. Corbett and J.L. Jensen: Immiscible Flow Behavior in Laminated and Cross-Bedded Sandstones in J. Petr. Science and Engineering, Vol. 9 (1993).

[9] P.W.M. Corbett, P.S. Ringrose, J.L. Jensen, and K.S. Sorbie: Laminated Clastic Reservoirs: The Interplay of Capillary Pressure and Sedimentary Architecture, paper SPE 24699 presented at the 67th SPE Annual Technical Conference, Washington D.C., Oct. 1992.

[10] Huang, Y., Ringrose, P.S., Sorbie, K.S., and Tudhope, S.W.: Waterflood Displacement Mechanisms in a Laminated Rock Slab: Validation of Predicted Capillary Trapping Mechanisms, paper 
SPE 28942 presented at the 69th SPE Annual Technical Conference, New Orleans, Sept. 1994.

[11] Angert, P.F . and Begg, S.H.: Modeling the Effect of Imbibition Capillary Pressure in Heterogeneous Porous Media: Waterflood Recoveries of the Romeo Interval, Prudhoe Bay Field, paper SPE 26081 presented at the Western Regional Meeting, Anchorage, May 1993.

[12] Fitzmorris, R.E., Kelsey, F.J., and Pande, K.K.: Effect of Crossflow on Sweep Efficiency in Water/Oil Displacement in Heterogeneous Reservoirs, paper SPE 24901 presented at the 67 th SPE Annual Technical Conference, Washington D.C., Oct. 1992.

[13] Holden L.: The Buckley-Leverett equation with spatially stochastic flux function, submitted 1994 to SIAM Journal on Applied Mathematics.

[14] Tjølsen, and Damsleth, E.: A Model for the Simultaneous Generation of Core-Controlled Stochastic Absolute and Relative Permeability Fields, paper SPE 22691 presented at the 66th SPE Annual Technical Conference, Dallas, Oct. 1991.

[15] Kristiansen, J.I., Mikkelsen, M. and Esbensen, K.: A Modified Leverett Approach and PLSRegression for Integrated Formation Evaluation, paper presented at the 33rd Annual Symposium of the Society of Professional Well Log Analysts, June 1992.

[16] Lantz, R.B.: Quantitative Evaluation of Numerical Diffusion (Truncation Error), Transactions of the SPE of the AIME, Vol. 251 (1971) $315-320$

\section{Table 1 Parameter statistics}

\begin{tabular}{lrrrrrr}
\hline l'ariable & $N$ & . Hean & St. dev. & . Hin. & Max. & Norm. * \\
\hline 0 & 41 & 0.214 & 0.022 & 0.151 & 0.248 & no \\
$k_{x}$ & 40 & 319 & 458 & 12 & 2823 & no \\
$\log _{e} k_{x}$ & 40 & 5.26 & 0.99 & 2.51 & 7.94 & \\
$S_{w i}$ & 41 & 0.221 & 0.074 & 0.070 & 0.488 & \\
$S_{n+w}$ & 41 & 0.266 & 0.049 & 0.200 & 0.392 & no \\
$n_{o}$ & 40 & 3.14 & 0.63 & 2.24 & 5.33 & no \\
$\log _{e} n_{o}$ & 40 & 1.11 & 0.19 & 0.81 & 1.67 & \\
$n_{t w}$ & 40 & 2.61 & 1.05 & 0.69 & 6.78 & no \\
$\log _{e} n_{w}$ & 40 & 0.89 & 0.37 & -0.37 & 1.91 & \\
$k_{r+w}\left(S_{o r}\right)$ & 41 & 0.39 & 0.15 & 0.06 & 0.69 & \\
\hline
\end{tabular}

" "No" denotes significant deviation from normal distr.

Table 2 Correlation coefficients

\begin{tabular}{lccrrrr}
\hline & $\log _{e} k_{H}$ & \multicolumn{1}{c}{$S_{w i}$} & \multicolumn{1}{c}{$S_{o r}$} & $\log _{e} n_{o}$ & $\log _{e} n_{w}$ & $k_{r w}\left(S_{o r}\right)$ \\
\hline 0 & 0.44 & -0.42 & -0.02 & -0.30 & 0.14 & 0.17 \\
$\log _{e} k_{H}$ & & -0.77 & 0.19 & 0.52 & -0.59 & 0.81 \\
$S_{w i}$ & & & -0.53 & -0.37 & 0.58 & -0.63 \\
$S_{\text {orw }}$ & & & & 0.21 & -0.35 & 0.32 \\
$\log _{e} n_{0}$ & & & & & -0.73 & 0.67 \\
$\log _{e} n_{w}$ & & & & & & -0.57 \\
\hline
\end{tabular}

bold face indicates strong correlation between variables.
Table 3 Run summaries

\begin{tabular}{|c|c|c|c|c|}
\hline \multirow{2}{*}{$\begin{array}{c}\text { Production } \\
\text { Characterities }\end{array}$} & \multicolumn{2}{|c|}{$\begin{array}{c}\text { Water Breakthrough } \\
\text { Years }\end{array}$} & \multicolumn{2}{|c|}{$\begin{array}{c}\text { Total Oil Production } \\
10^{6} \mathrm{Sm}^{3}\end{array}$} \\
\hline & $\begin{array}{c}\text { Grid } \\
180 \times 48\end{array}$ & $\begin{array}{r}\text { Grid } \\
36 \times 48\end{array}$ & $\begin{array}{c}\text { Grid } \\
180 \times 48\end{array}$ & $\begin{array}{l}\text { Grid } \\
36 \times 48\end{array}$ \\
\hline $\begin{array}{lcc}\text { Const.rel.perm.: } & \\
\Delta \rho=0, & \text { wo } / P_{c}, & . \mathrm{H}=1 \\
\Delta \rho=0, & \text { w } / P_{c}, & . \mathrm{H}=1 \\
\Delta \rho=0, & \text { wo } / P_{c}, & \mathrm{H}=10 \\
\Delta \rho=0 . & \text { wi } P_{c}, & \mathrm{H}=10\end{array}$ & $\begin{array}{l}5.51 \\
5.61 \\
0.93 \\
0.95 \\
\end{array}$ & $\begin{array}{l}4.74 \\
4.96 \\
0.65 \\
0.66 \\
\end{array}$ & $\begin{array}{l}2.745 \\
2.802 \\
1.820 \\
1.853\end{array}$ & $\begin{array}{l}2.708 \\
2.781 \\
1.786 \\
1.837\end{array}$ \\
\hline $\begin{array}{lcc}\text { Var.rel.perm.: } \\
\Delta \rho=0, \\
\Delta \rho=0, \quad \text { wo } / P_{c}, & M=1 \\
\Delta \rho=0, & \text { wo } / P_{c}, & M=10 \\
\Delta \rho=1 & \\
\Delta \rho=0, & \text { w } / P_{c} . & M=10\end{array}$ & $\begin{array}{l}5.12 \\
3.59 \\
1.05 \\
0.99\end{array}$ & $\begin{array}{l}2.82 \\
2.60 \\
0.27 \\
0.84\end{array}$ & $\begin{array}{l}2.340 \\
2.363 \\
1.646 \\
1.671\end{array}$ & $\begin{array}{l}2.353 \\
2.362 \\
1.585 \\
1.613\end{array}$ \\
\hline
\end{tabular}

\begin{tabular}{|c|c|c|c|c|}
\hline \multirow{2}{*}{$\begin{array}{l}\text { Production } \\
\text { Characterities }\end{array}$} & \multicolumn{2}{|c|}{$\begin{array}{c}\text { Water Breakthrough } \\
\text { Years }\end{array}$} & \multicolumn{2}{|c|}{\begin{tabular}{|c|} 
Total Oil Production \\
$10^{\circ} \mathrm{Sm}^{3}$
\end{tabular}} \\
\hline & \begin{tabular}{|c|} 
Grid \\
$36 \times 240$ \\
\end{tabular} & $\begin{array}{r}\text { Grid } \\
36 \times 48 \\
\end{array}$ & \begin{tabular}{|c} 
Grid \\
$36 \times 240$ \\
\end{tabular} & $\begin{array}{r}\text { Grid } \\
36 x+8 \\
\end{array}$ \\
\hline Const.rel.perm.: & & & & \\
\hline$\Delta \rho \neq 0$, wo $/ P_{e}, \quad \mathrm{~B}=1$ & 3.11 & 3.03 & 2.865 & 2860 \\
\hline$\Delta \rho \neq 0, \quad w / P_{\varepsilon}, \quad u=1$ & 3.21 & 3.21 & 2.860 & 2.857 \\
\hline$\Delta \rho \neq 0$, wo $/ P_{s}, \quad M=10$ & 1.48 & 1.27 & 1.738 & 1.897 \\
\hline$\Delta \rho \neq 0 . \quad w / P, \quad M=10$ & 1.60 & 1.22 & 1.896 & 1.899 \\
\hline Var.rel.perm.: & & & & \\
\hline$\Delta \rho \neq 0$, wo $/ P_{6}, \quad M=1$ & 2.95 & 2.96 & 2.688 & 2.683 \\
\hline$\Delta \rho \neq 0, \quad w / P_{s}, \quad M=1$ & 1.83 & 2.39 & 2.514 & 2.549 \\
\hline$\Delta \rho \neq 0$, wo $/ P_{\epsilon}, \quad M=10$ & 1.51 & 1.17 & 1.622 & 1.738 \\
\hline$\Delta \rho \neq 0 . \quad w / P_{c}, \quad M=10$ & 0.84 & 1.22 & 1.586 & 1.655 \\
\hline
\end{tabular}

Fig.1 Horizontal permeabilities, Grid $36 \times 48$

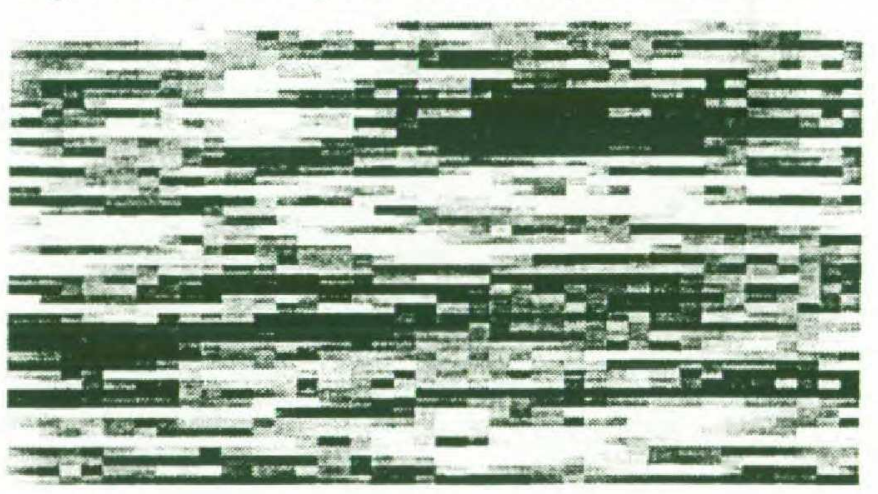

Fig.2 Effect of cap.pres. on oil production profile; no gravity; grid $180 \times 48$ (no cap.pres. - , with cap.press. ...)

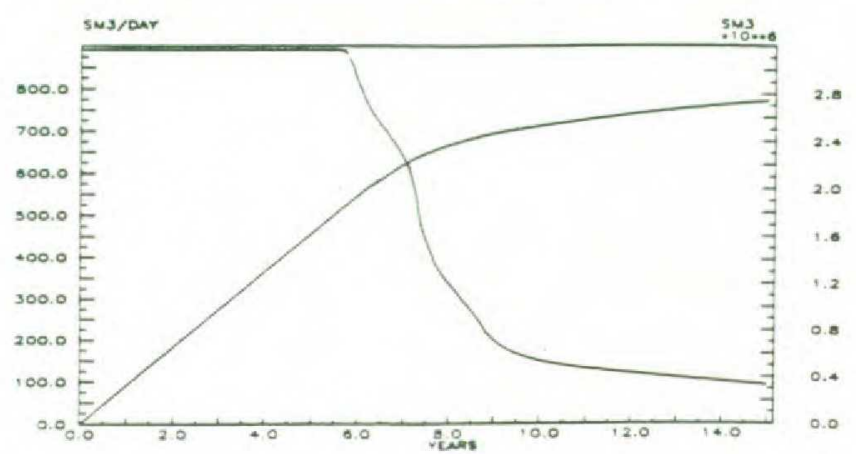

Fig. 2a Constant $k_{r}, P_{c} ; M=1$ 


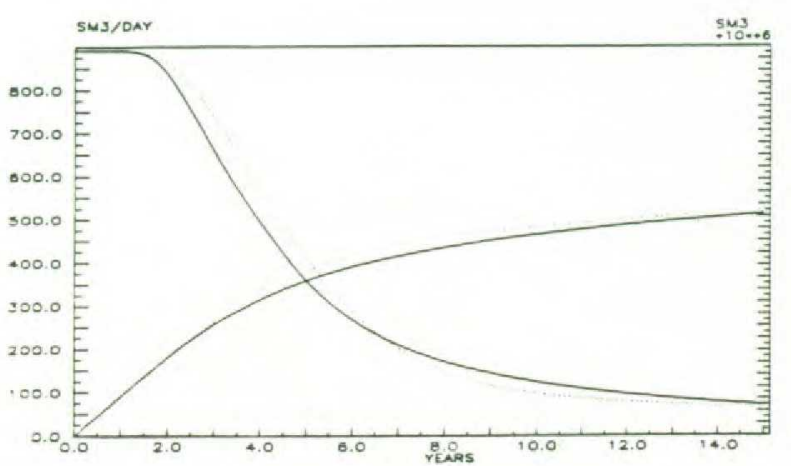

Fig. 2b Constant $k_{r}, P_{c} ; M=10$

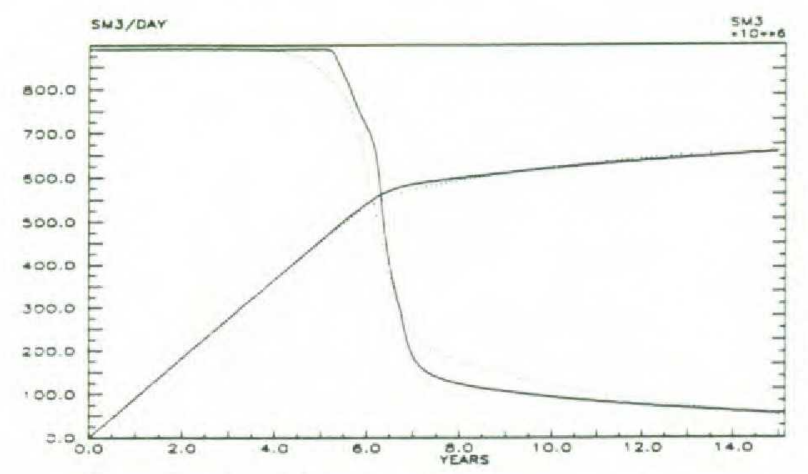

Fig. 2c Variable $k_{r}, P_{c} ; M=1$

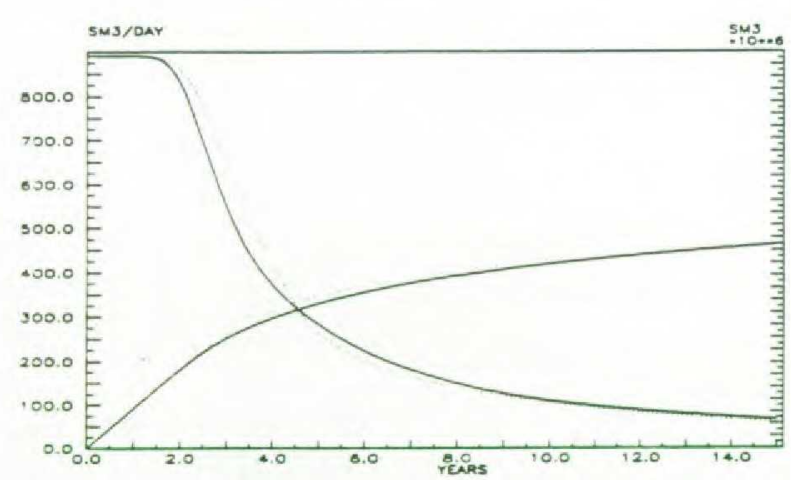

Fig. 2d Variable $k_{r}, P_{c} ; M=10$

Fig.3 Effect of cap.pres. on saturation distribution; no gravity; grid $180 \times 48$; variable $k_{r} ; M=10$

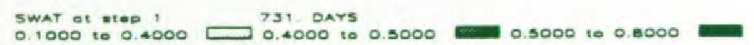

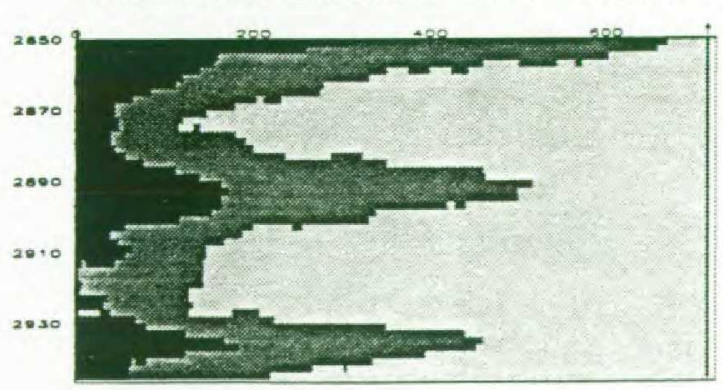

Fig. 3a Saturation after 2 years; $P_{c}=0$

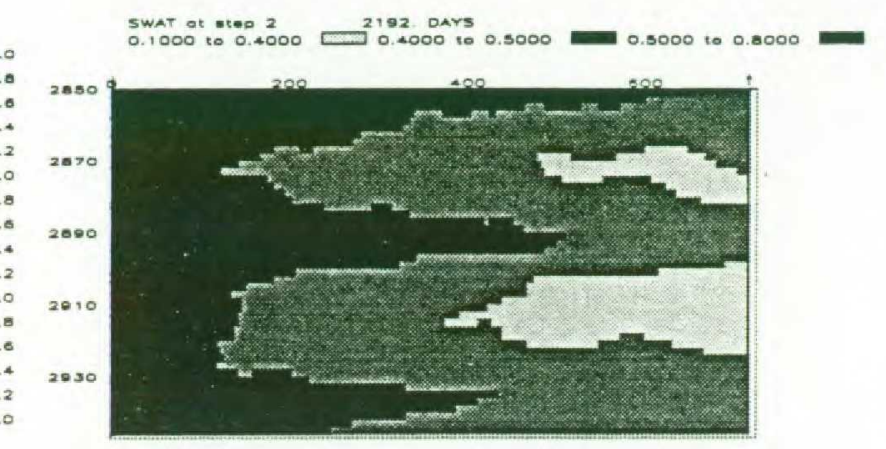

Fig. 3b Saturation after 6 years; $P_{c}=0$

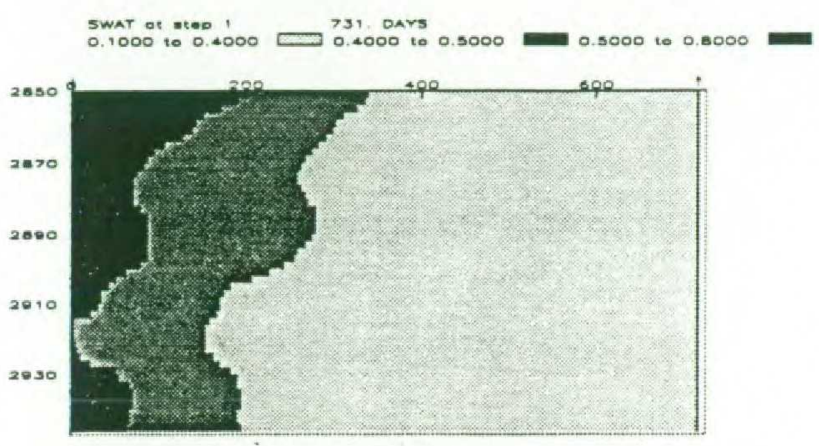

Fig. $3 c$ Saturation after 2 years; $P_{c}>0$

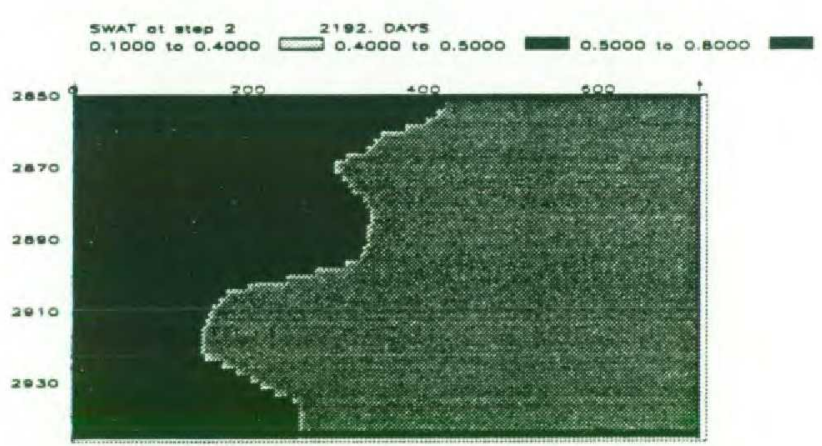

Fig. 3d Saturation after 6 years; $P_{c}>0$

Fig. 4 Effect of const./var. cap.pres on saturation distribution; no gravity; grid 180 $\times 48 ; M=1$

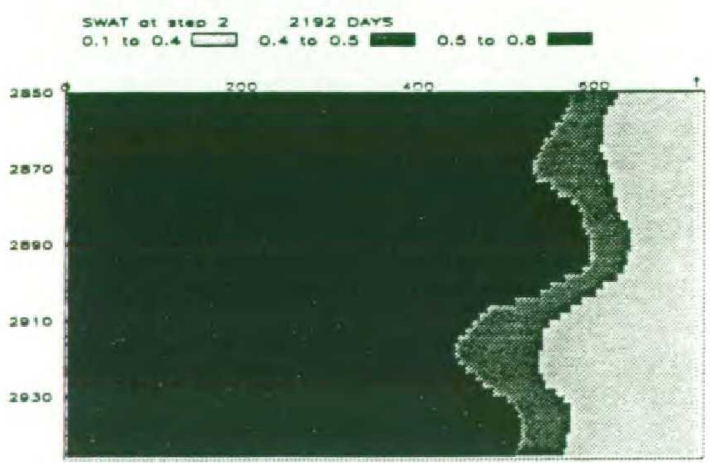

Fig. 4a Saturation after 6 years; constant $k_{r}, P_{c}$ 
SWAT Ot M1002 2192 DANS

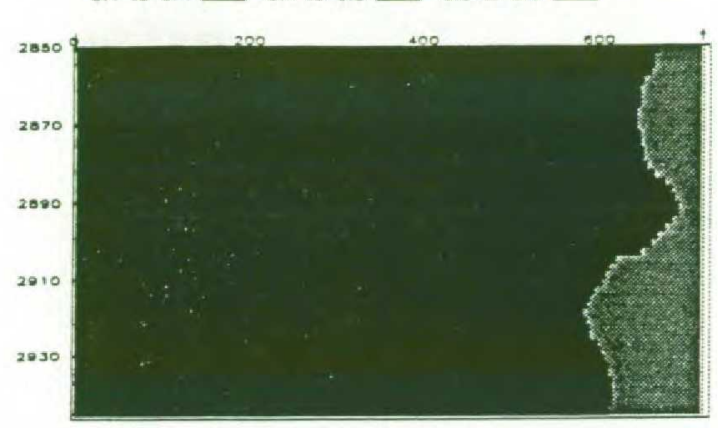

Fig. 4b Saturation after 6 years; variable $k_{r}, P_{c}$

Fig.5 Effect of cap.pres. on oil production profile; with gravity; grid $36 \times 240$ (no cap.pres. - , with cap.press. ...)

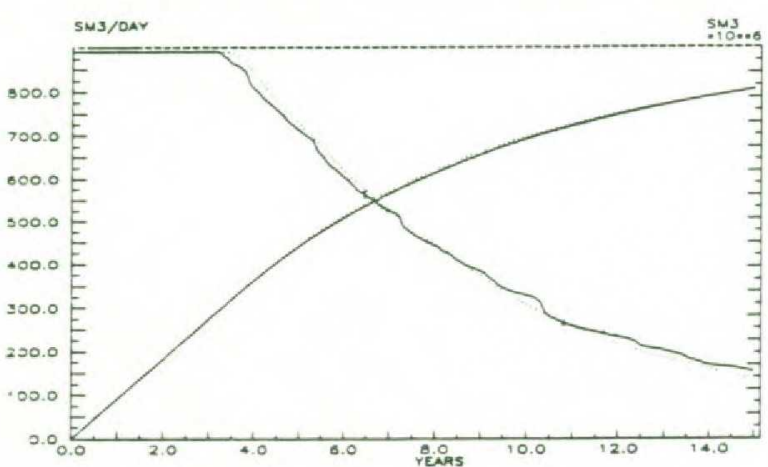

Fig. 5a Constant $k_{r}, P_{c} ; M=1$

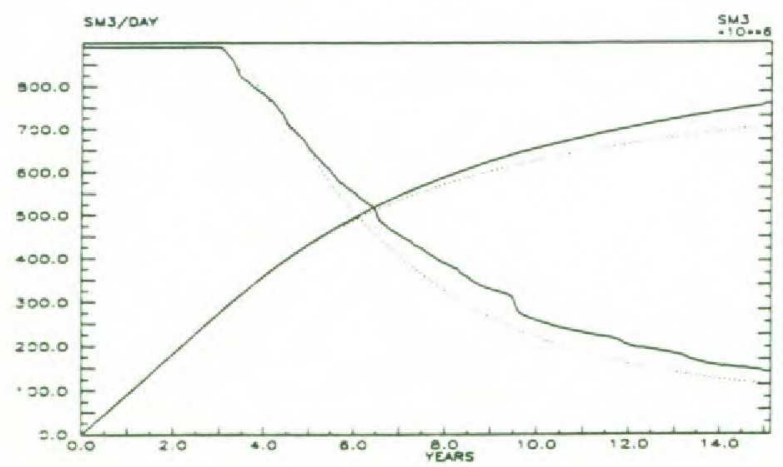

Fig. 5b Variable $k_{r}, P_{c} ; M=1$

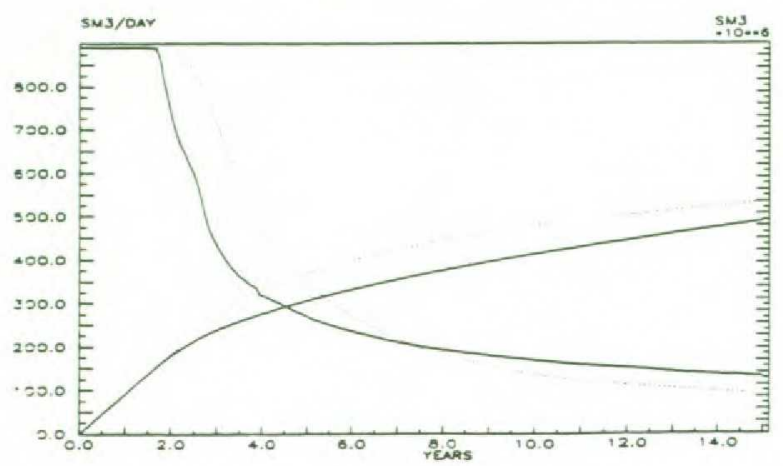

Fig. 5c Constant $k_{r}, P_{c} ; M=10$

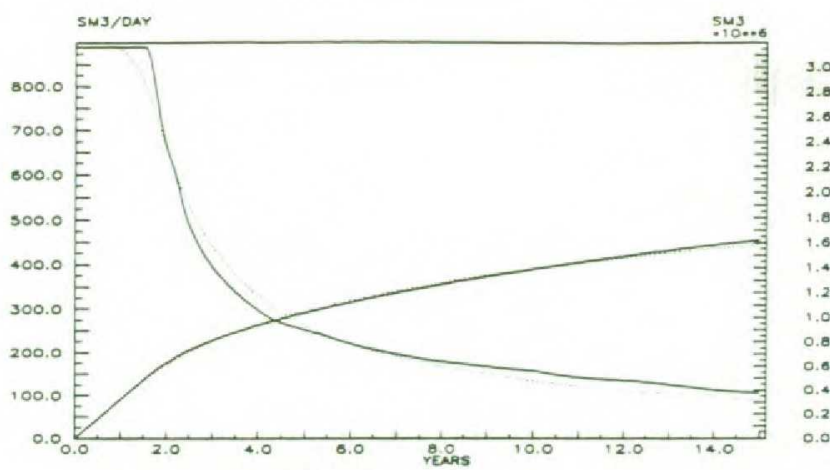

Fig. 5d Variable $k_{r}, P_{c} ; M=10$

Fig. 6 Effect of no/const. cap.pres on saturation distribution; with gravity; grid 36 $\times 240 ; M=10$

SWAT at steP $1 \quad$ 731. DAYS

CONTOUR INTERVAL 0.1000

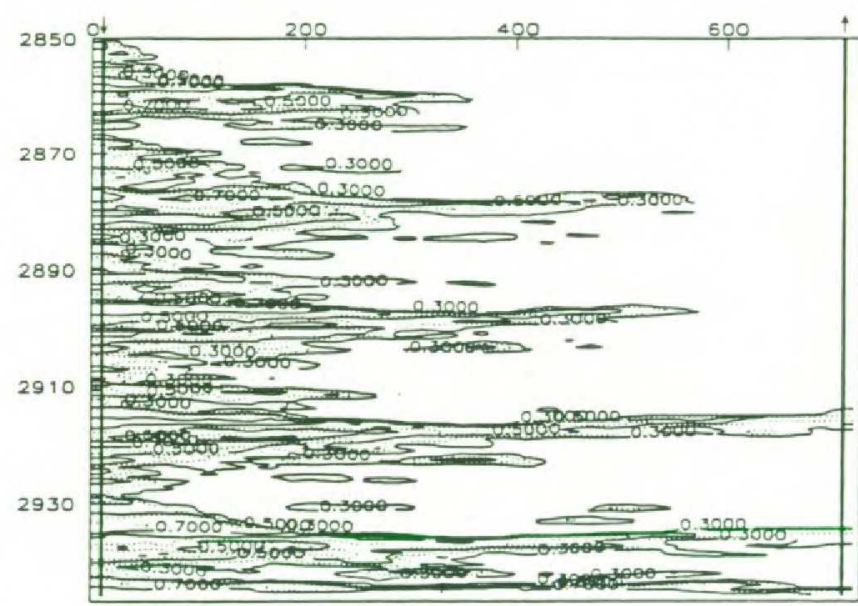

Fig. 6a Saturation after 2 years; $P_{c}=0$

$\begin{array}{ll}\text { SWAT Ot STEP } 2 & \text { 2192. DAYS } \\ \text { CONTOUR INTERVAL } & 0.1000\end{array}$

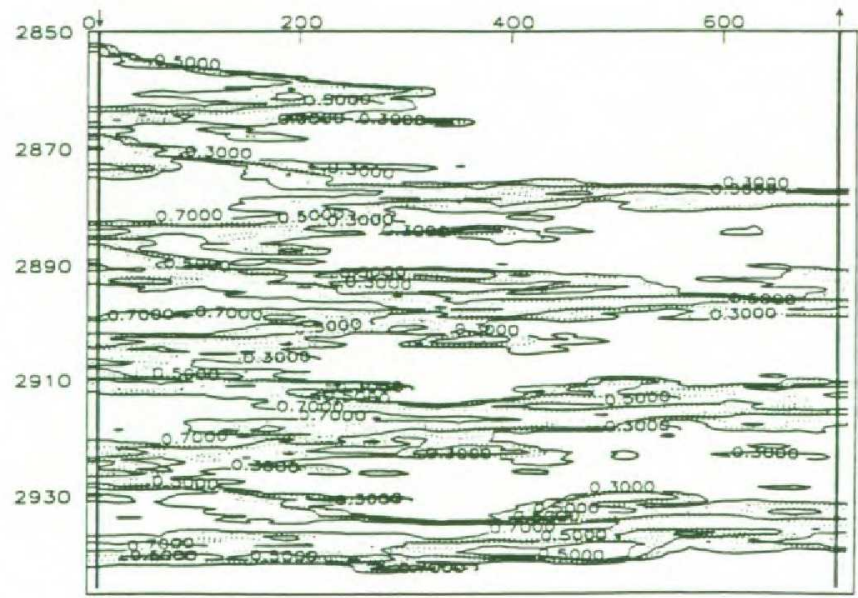

Fig. 6b Saturation after 6 years; $P_{c}=0$ 
SWAT Ot step I

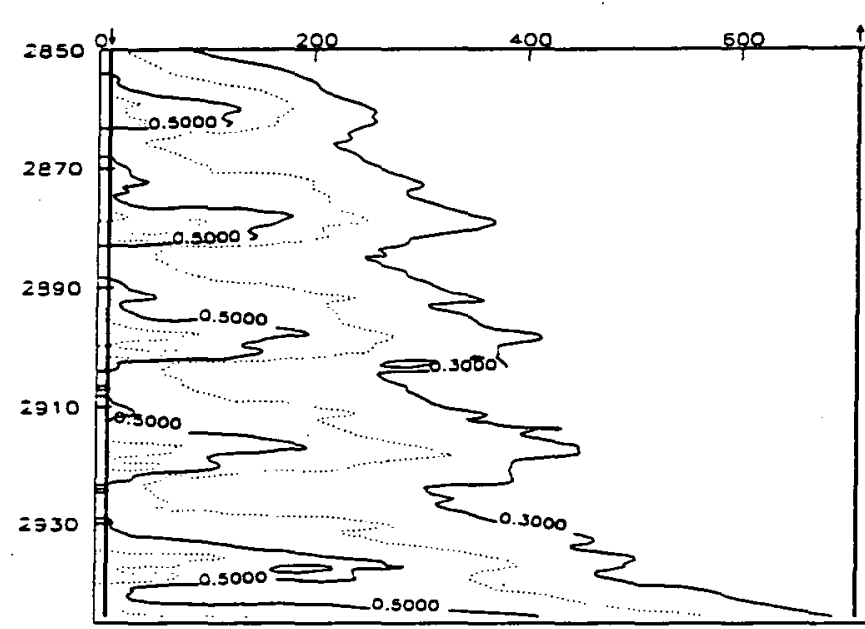

Fig. 6c Saturation after 2 years; constant $k_{r}, P_{c}$

SWAT OT STED 2192 . DAYS

CONTOUR INTERVAL 0.1000

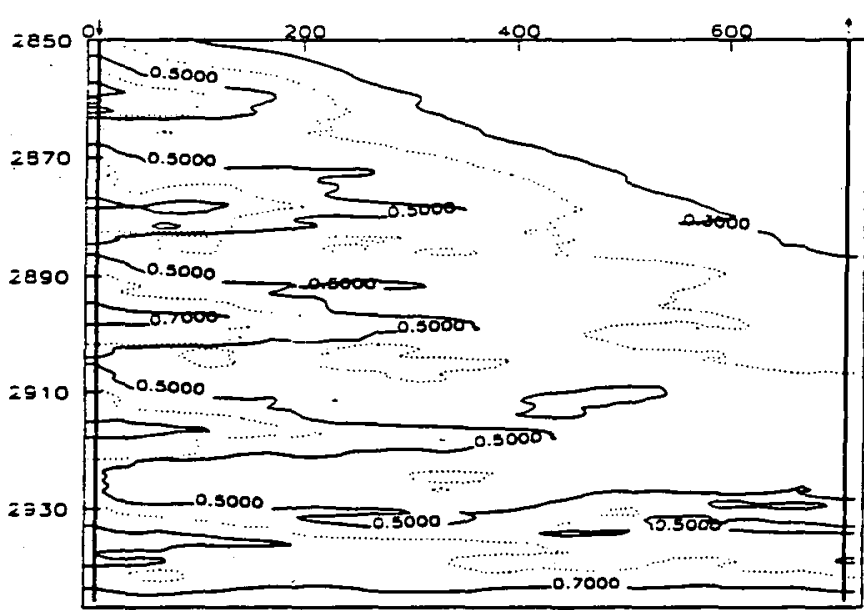

Fig. 6d Saturation after 6 years; constant $k_{r}, P_{c}$

Fig.7 Five realizations; with gravity; grid $36 \times$ 240; $M=10$

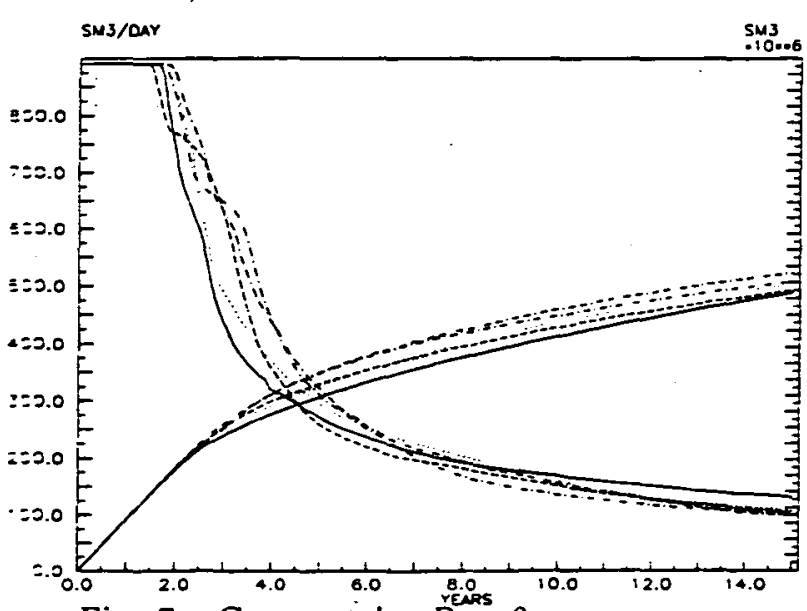

Fig. 7a Constant $k_{r} ; \stackrel{P_{c}}{\text { cans }}=0$

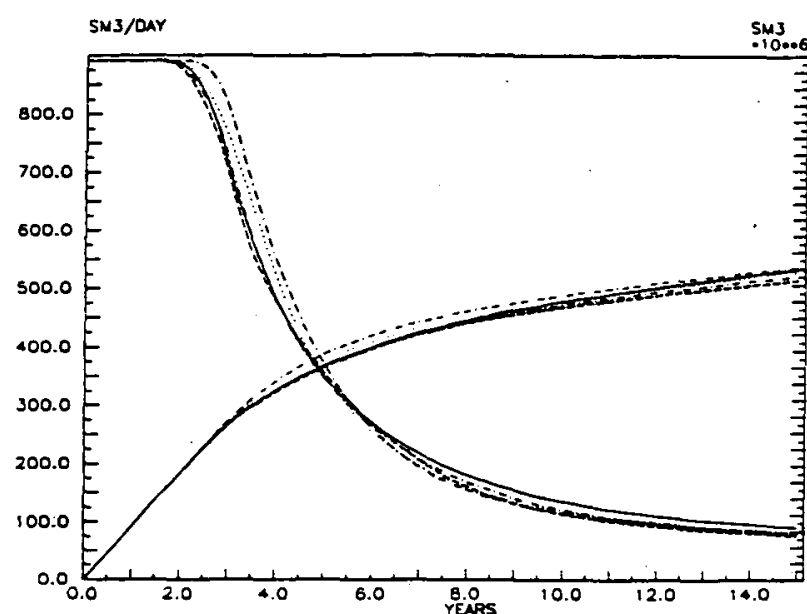

Fig. $7 \mathrm{~b}$ Constant $k_{r} ; P_{c}>0$

Fig.8 Five realizations; no gravity; grid $180 \times$ 48; $M=1$

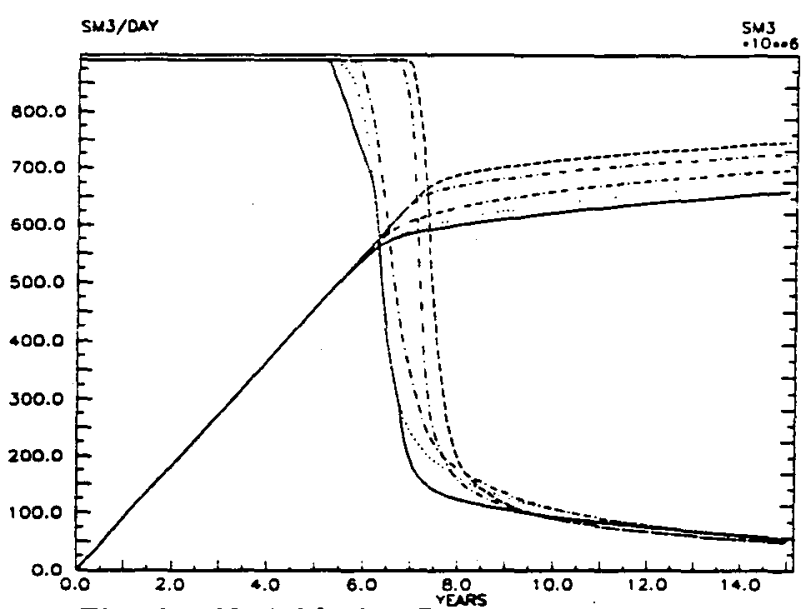

103

Fig. 8a Variable $k_{r} ; P_{c}=0$

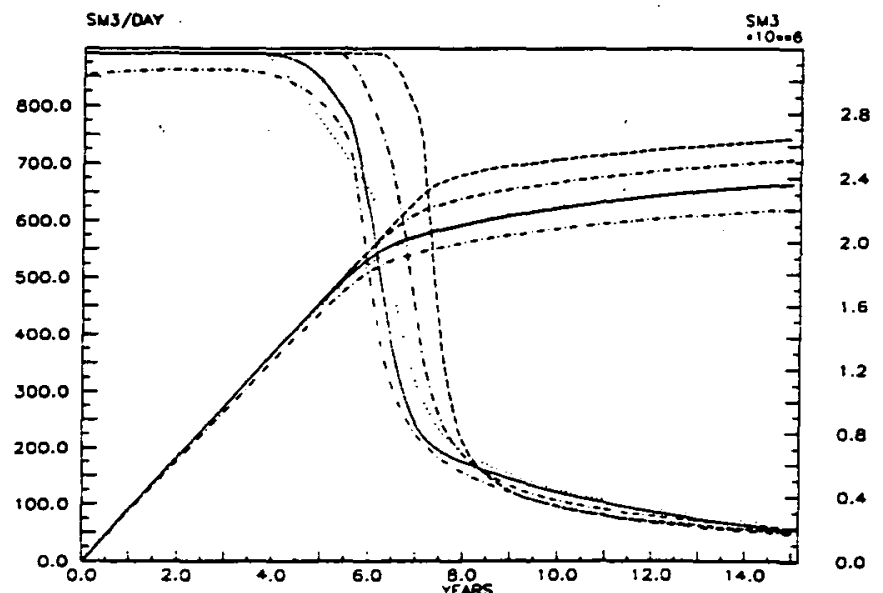

Fig. 8b Variable $k_{r} ; P_{c}>0$ 
Fig.9 Comparison of fine and coarse grid in the $x$-direction; no gravity; variable $k_{r}$, (grid $180 \times 48$ - , grid $36 \times 48 \cdots$ )

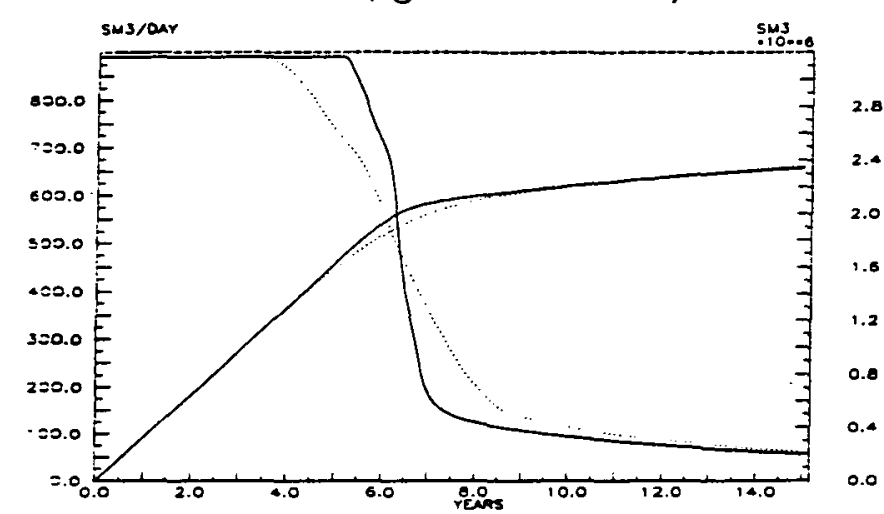

Fig. 9a Variable $k_{r} ; P_{c}=0 ; M=1$
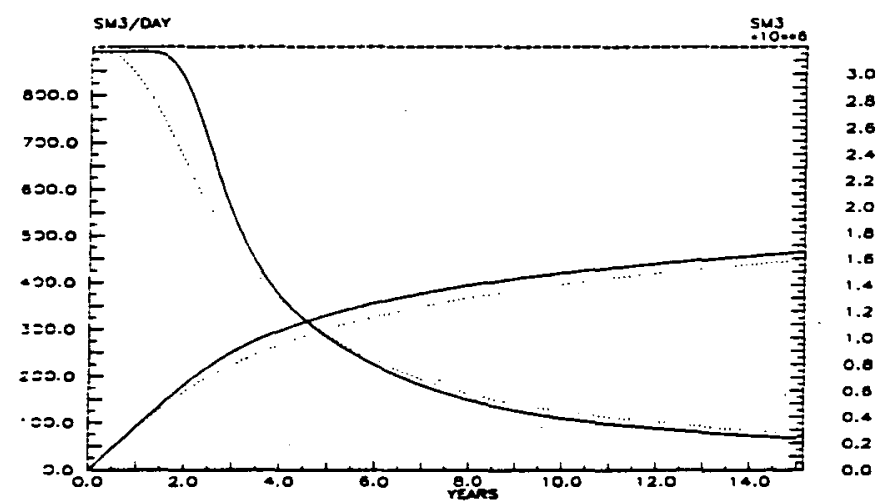

Fig. 9b Variable $k_{r} ; P_{c}=0 ; M=10$

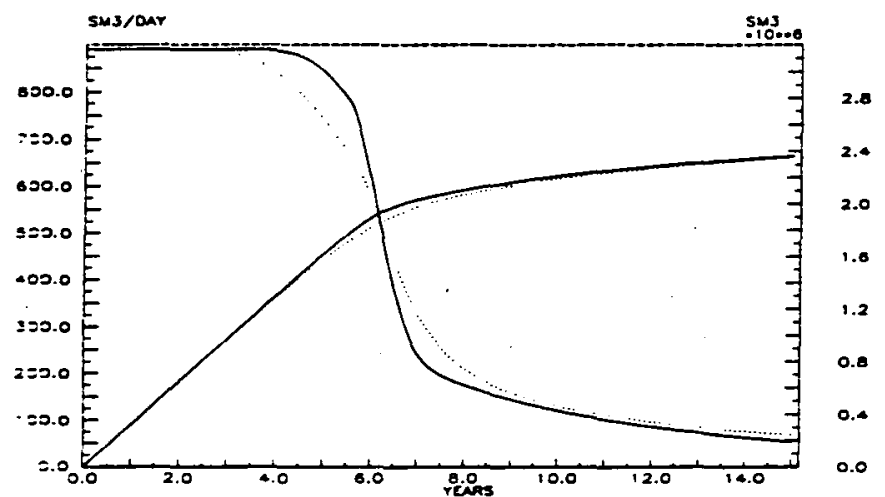

Fig. 9c Variable $k_{r}, P_{c} ; M=10$

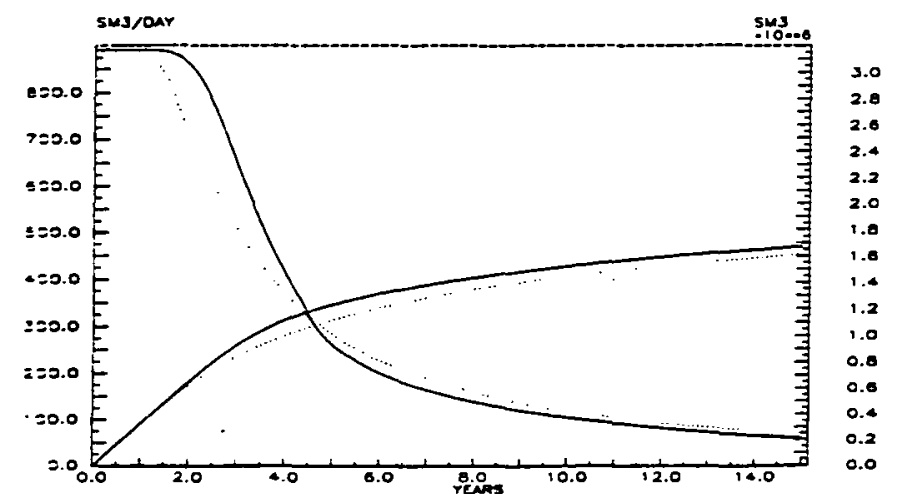

Fig. 9d Variable $k_{r}, P_{c} ; M=10$ 